\title{
Gene-Eden-VIR Is Antiviral: Results of a Post Marketing Clinical Study
}

\author{
Hanan Polansky ${ }^{*}$ Edan Itzkovitz \\ The Center for the Biology of Chronic Disease (CBCD), Rochester, USA. \\ Email: "hpolansky@cbcd.net
}

Received July $3^{\text {rd }}, 2013$; revised August $3^{\text {rd }}, 2013$; accepted August $12^{\text {th }}, 2013$

Copyright (C) 2013 Hanan Polansky, Edan Itzkovitz. This is an open access article distributed under the Creative Commons Attribution License, which permits unrestricted use, distribution, and reproduction in any medium, provided the original work is properly cited.

\begin{abstract}
Introduction: This paper reports the results of a post marketing clinical study that tested the antiviral properties of Gene-Eden-VIR ${ }^{\mathrm{TM}}$. Specifically, the clinical study tested the effect of Gene-Eden-VIR on the severity, duration, and frequency of symptoms reported by individuals infected with various viruses. The viruses included the Human Papillomavirus (HPV), Herpes Simplex Virus (HSV), Epstein Barr Virus (EBV), Human Cytomegalovirus (HCMV) and Hepatitis C Virus (HCV). The symptoms included abnormal Pap smear, low and high grade cervical dysplasia, warts, blisters, cold sores, hives, skin tabs, panic attacks, depression, kidney problems, sleeping problems, liver problems, fever, fatigue, sore throat, swollen lymph nodes, diarrhea, and weight loss. Treatment: A capsule of Gene-Eden-VIR includes five natural ingredients: $100 \mathrm{mg}$ of quercetin, $150 \mathrm{mg}$ of green tea extract, $50 \mathrm{mg}$ of a cinnamon extract, $25 \mathrm{mg}$ of a licorice extract, and $100 \mathrm{mcg}$ of selenium. The dosage was 1, 2, 3, or 4 capsules per day. The duration of treatment was 2 to 54 weeks. Population: The study population consisted of 60 infected individuals, ages 20 to 66. Results: The participants reported no side effects after taking Gene-Eden-VIR. Seventy three percent of the individuals treated with Gene-Eden-VIR reported a decrease in their symptoms. Specifically, they reported a decrease in the severity $(p=0.006$, $\mathrm{n}=45)$, duration $(\mathrm{p}=0.009, \mathrm{n}=34)$, and frequency of their symptoms $(\mathrm{p}<0.001, \mathrm{n}=31)$. Following treatment, the participants also reported an increase in their physical abilities $(\mathrm{p}<0.001, \mathrm{n}=47)$, energy levels $(\mathrm{p}<0.001, \mathrm{n}=54)$, mental abilities $(\mathrm{p}<0.001, \mathrm{n}=44)$, and general health $(\mathrm{p}<0.001, \mathrm{n}=46)$. The results showed that Gene-Eden-VIR has a duration effect $(\mathrm{p}=0.044, \mathrm{n}=32)$, that is, those treated for a longer time reported a larger decrease in their symptoms. The results showed no interviewer bias, no selection bias, and a surprising response shift. The results also showed that Gene-Eden-VIR has therapeutic consistency under varying manufacturing conditions. Conclusions: This post marketing clinical study showed that Gene-Eden-VIR is a safe and effective antiviral treatment. Specifically, the clinical study showed that Gene-Eden-VIR is a safe and effective treatment against the Human Papillomavirus (HPV), Herpes Simplex Virus (HSV), Epstein Barr Virus (EBV), Human Cytomegalovirus (HCMV), and Hepatitis C Virus (HCV). Therefore, health care practitioners should recommend Gene-Eden-VIR as a safe and effective antiviral treatment against these viruses.
\end{abstract}

Keywords: Human Papillomavirus (HPV); Herpes Simplex Virus (HSV); Epstein Barr Virus (EBV); Human Cytomegalovirus (HCMV); Hepatitis C Virus (HCV); Gene-Eden-VIR

\section{Introduction}

This paper reports the results of a post marketing clinical study that tested the antiviral properties of Gene-EdenVIR. Specifically, the clinical study tested the effect of Gene-Eden-VIR on the severity, duration, and frequency of symptoms in individuals infected with the Human Papillomavirus (HPV), Epstein Barr Virus (EBV), Herpes Simplex Virus (HSV), Human Cytomegalovirus

${ }^{*}$ Corresponding author.
(HCMV), and Hepatitis C Virus (HCV).

The following section describes the standard treatments against these viruses and notes their limitations.

HPV: There are no drugs approved against the HPV [1]. Current treatments include procedures, such as cryotherapy, conization, and the Loop Electrosurgical Excision Procedure (LEEP). These procedures use liquid nitrogen, a surgical knife (scalpel), a carbon dioxide $\left(\mathrm{CO}_{2}\right)$ laser, or electrical current to remove the abnormal growths caused by the HPV. These growths include cells 
that harbor the active virus. The procedures do not target cells with the latent virus. Since they do not remove the latent virus, these procedures only produce a temporary remission.

EBV: A few antiviral drugs are available that were shown to inhibit EBV replication in cell culture. These drugs include the acyclic nucleoside analogues aciclovir, ganciclovir, penciclovir, and their respective prodrugs valaciclovir, valganciclovir and famciclovir, the acyclic nucleotide analogues cidofovir and adefovir, and the pyrophosphate analogue foscarnet. However, clinical studies have shown that these drugs are mostly ineffective in humans [2].

HSV: Two types of antiviral treatments against HSV are available: topical and oral. The treatments include penciclovir, acyclovir, famciclovir, and valaciclovir. However, their effectiveness is limited. For instance, a metaanalysis of five placebo-controlled and two dosecomparison studies evaluated the effect of aciclovir, famciclovir or valaciclovir on symptoms. The meta-analysis showed that oral antiviral therapy decreases the duration and the associated pain of an outbreak by merely one day [3].

HCMV: Several drugs are approved for the treatment of HCMV infections in immunocompromised individuals. These drugs include ganciclovir, its oral prodrug valganciclovir, cidofovir, foscavir and fomivirsen. However, the use of these drugs in immunocompetent individuals is limited by their toxicity, poor oral bioavailability, modest efficacy, and the development of drug resistance [4].

HCV: The combination of a pegylated interferon (IFN)- $\alpha$ and ribavirin is the standard treatment for chronic HCV infections. This combination is effective in about $80 \%$ of the individuals infected with the HCV genotype 2 or 3 , and in about $40 \%-50 \%$ in those infected with genotype 1 or 4 . Lately, two new drugs were approved, telaprevir and boceprevir, with better results. However, the combinations of pegylated interferon (IFN)- $\alpha$ and ribavirin and telaprevir or boceprevir are associated with additional side effects, increased costs, and more complex treatment strategies [5].

There are also some dietary supplements that claim to be effective against viruses. However, the law does not consider dietary supplements as drugs, and therefore, does not require the FDA to evaluate the effectiveness of these supplements [6]. As a result, the sellers of most dietary supplements do not conduct clinical studies to test the effectiveness of their products.

The scientists, who developed Gene-Eden-VIR, used a unique scientific tool, Computer Intuition, a proprietary psycholinguistic-based, data-mining program that analyzes scientific text [7]. The scientists' objective was to identify natural ingredients mentioned in the scientific literature that have a strong antiviral effect against the most common viruses. To achieve the objective, they used Computer Intuition to analyze more than 50,000 papers. Based on the computer's results, they selected five ingredients: green tea extract, quercetin, licorice extract, cinnamon extract, and selenium.

A manual search on these five ingredients found studies that supported the computer's results. For instance, some studies showed that catechins, found in green tea, are effective against viruses such as Epstein-Barr Virus (EBV), Herpes Simplex Virus (HSV), Hepatitis Virus B (HVB), and other viruses [8-11]. Other studies showed that quercetin inhibits EBV-EA activation in latently infected cells [12-14]. Some studies showed that glycyrrhizin and glycyrrhizic acid, found in licorice, have an antiviral effect [15-18]. A few studies showed that the active compounds in cinnamon, cinnamaldehyde, terpenoids, eugenol, and ethyl cinnamate, have a strong antiviral effect [19-21]. Finally, some studies reported that selenium also has an antiviral effect [22-24].

After selecting the five ingredients, the scientists used Computer Intuition again to analyze the thousands of papers published on these five ingredients. Table 1 lists the number of scientific papers published on each ingredient according to PubMed as of September 1, 2009 (Table 1).

Based on the new computer's results, the scientists determined the final formula of Gene-Eden-VIR: quercetin $100 \mathrm{mg}$, green tea extract $150 \mathrm{mg}$, cinnamon extract 50 $\mathrm{mg}$, selenium $100 \mathrm{mcg}$, and licorice extract $25 \mathrm{mg}$. GeneEden-VIR was introduced in the marketplace at the end of 2009.

This paper reports the results of a post-marketing clinical study that tested the antiviral properties of GeneEden-VIR. Specifically it tested the effect of Gene-EdenVIR on the severity, duration, and frequency of symptoms reported by individuals infected with the HPV, EBV, HSV, HCMV, and HCV.

\section{Methods}

\subsection{Ethics Statement}

An informed consent was obtained from all participants

Table 1. Scientific papers per ingredient on PubMed.

\begin{tabular}{cc}
\hline Ingredient & Number of Scientific Papers \\
\hline Green Tea Extract & 3413 \\
Quercetin & 6753 \\
Licorice Extract & 2215 \\
Cinnamon Extract & 913 \\
Selenium & 2004 \\
Total & $\mathbf{1 5 , 2 9 8}$ \\
\hline
\end{tabular}


prior to conducting the phone interviews.

\subsection{Objective and Framework}

We used this post marketing study to test the efficacy, safety, and optimal use of Gene-Eden-VIR during viral infections. Specifically, we tested the effect of GeneEden-VIR on the severity, duration, and frequency of symptoms reported by individuals infected with the HPV, EBV, HSV, HCMV, and HCV.

\subsection{Treatment}

The dosage was 1, 2, 3, or 4 capsules of Gene-Eden-VIR per day. The duration of treatment ranged from 2 to 54 weeks.

\subsection{Outcome Measures}

We used a self-developed questionnaire called the Natural Origin Treatment Clinical Questionnaire (NotCiq). The NotCiq questionnaire is a patient reported outcome (PRO) instrument. The purpose of a PRO instrument is to capture the patient's experience. Our main endpoint was symptoms associated with viral infections. Meaning, the objective of the study was to measure the effect of the treatment with Gene-Eden-VIR on the symptoms of the viral infection as they are reported by the treated participants.

To develop a reliable and valid questionnaire, we considered the purpose of study, the research question, the response format, the phrasing of tested items, and the statistical tests. At the end, we created a five section questionnaire. The first section measured the changes in general health. A second section centered on the changes in the severity, duration, and frequency of the symptoms during a viral infection. A third section centered on changes in physical abilities, a fourth on energy, and a fifth on mental abilities. The general health section included 5 questions. The section explored areas such as current disease and the change in general health. The symptoms section included 5 questions, two questions on severity, two questions on duration, and one question on the frequency of symptoms. The physical abilities section included 5 questions, the energy section included 5 questions, and the mental abilities section included 6 questions. NotCiq included both open and closed-ended questions. The answers to the closed-ended questions were on a scale of 1 to 7 , where " 1 " corresponded to "Poor health", "Very Severe", "Extremely Interfered", "All the time", "No relief", or "Frequently appeared", and 7 to "Unnoticeable", "Not at All", "Constant Relief", or "Never Appeared", etc.

The study collected the answers to the NotCiq instrument by phone interviews. We used two independent companies that specialized in outbound call services for performing the interviewers, one company from the US and one from Israel. The interviewers were blinded to the objective of the study. All interviews were recorded.

The instrument was pre-tested on a small sample of Gene-Eden-VIR users to evaluate both the sensitivity and clarity of the questions.

\subsection{Population}

We randomly selected participants from the Gene-Eden VIR customer database that includes all Gene-Eden-VIR current and past customers. We used a computerized system to randomly create a call list of 1000 customers. Out of these customers, 100 agreed to participate. From these participants we excluded customers who were using Gene-Eden-VIR for other purposes, such as treatment for cancer, chronic diseases, hypertension, etc. The final list of participants consisted of 60 Americans of both sexes, ages 20 to 66, infected with the HPV, EBV, HSV, HCMV, and HCV. The diagnosis was done by the participant's physician. Each participant reported as having specific symptoms (e.g. for HPV participants reported genital warts, low and high grade cervical dysplasia, abnormal Pap smear results) and general symptoms (blisters, cold sores, hives, skin tabs, panic attacks, depression, kidney problems, sleeping problems, liver problems, fever, fatigue, sore throat, swollen lymph nodes, diarrhea, and weight loss). Since the objective of the study was to test the effect of Gene-Eden-VIR on symptoms associated with viral infections, we excluded participants who reported no symptoms. That is, we excluded participants who reported a 7 point score on the pre-treatment question. Such a score indicates that the participant does not suffer from the symptom represented by the question regardless of the treatment the participant actually received. This exclusion still preserves the intention to treat (ITT) principle.

We considered participants who stopped taking GeneEden-VIR for a month or more before data collection as past users. All other participants were considered as present users.

\subsection{Controls}

The Gene-Eden-VIR post marketing study includes a pre-treatment concurrent control and an historical control. To create an historical control, we divided the original test group into two subgroups, present users and past users. Generally, an historical control is a separate group. However, since we did not have a separate group of non-users, we used the past users as a proxy for an historical control.

\subsection{Statistical Analysis}

We tested the statistical difference between the score of 
pre-treatment, which is the numeric answer each participant used to describe his symptoms before the treatment started, to the score of post-treatment, which is the numeric answer each participant used to describe his symptoms after the treatment was completed. We also calculated the delta $(\Delta)$, that is, the difference in scores between the answer to the pre-treatment and post-treatment question. Then, we tested the statistical difference between the deltas. These tests were performed in a thentest model for both present and past users. Statistical analysis was performed using a two-tail t-test assuming unequal variances.

The research defined the primary endpoint as a statistically significant increase in the score from pre-treatment to post-treatment on the raw answers and on the deltas.

\section{Results}

The participants reported no side effects after taking Gene-Eden-VIR.

Out of the 60 infected participants, 7 reported perfect general health and no symptoms (that is, a score 7 out of 7 on the pre-treatment questions on both the general health question and the symptoms questions), and 7 participants reported perfect general health with some symptoms. Fifty four percent (25/46) of the individuals that reported less then perfect health reported an improvement in general health.

Out of the 60 infected participants, 41 reported having some symptoms. Seventy three percent $(30 / 41)$ of the individuals treated with Gene-Eden-VIR reported a decrease in their symptoms. Specifically, they reported a decrease in the severity of their symptoms $(p=0.006, n=$ $45)$, a decrease in the duration of their symptoms ( $p=$ $0.009, \mathrm{n}=34)$, and a decrease in the frequency of their symptoms $(\mathrm{p}<0.001, \mathrm{n}=31)$ (Table 2).

Although $73 \%$ of the participants with symptoms reported a decrease in their symptoms, only $54 \%$ reported an improvement in their general health. These numbers

Table 2. Pre-treatment vs. post-treatment symptoms as reported by the participants.

\begin{tabular}{ccccc}
\hline Questions & N of $\mathbf{P}^{* \#}$ & $\begin{array}{c}\text { Pre-T } \\
\text { Score }\end{array}$ & $\begin{array}{c}\text { Post-T } \\
\text { Score }\end{array}$ & P Value \\
\hline A5 General Health & 46 & 4.67 & 5.59 & $<0.001$ \\
B1-B2 Severity & 45 & 4.90 & 5.49 & 0.006 \\
B3-B4 Duration & 34 & 4.79 & 5.46 & 0.009 \\
B5 Frequency & 31 & 2.42 & 5.23 & $<0.001$ \\
\hline
\end{tabular}

${ }^{*}$ The statistical analysis on the Severity of Symptoms, and on the Duration of Symptoms, was conducted using one score per participant. The score was equal to the average answers of the participant to questions B1 and B2, and $\mathrm{B} 3$ and B4, respectively. ${ }^{\#} \mathrm{~N}$ of $\mathrm{P}$ is Number of Participants, Pre-T is Pre Treatment, Post-T is Post Treatment. suggest that some participants do not perceive a decrease in symptoms associated with viral infections as an improvement in general health. In their mind, viral symptoms and general health are somewhat unrelated (Table 2).

Following treatment with Gene-Eden-VIR, the participants also reported an increase in their physical abilities $(\mathrm{p}<0.001, \mathrm{n}=47)$, an increase in their energy levels $(\mathrm{p}<0.001, \mathrm{n}=54)$, and an increase in their mental abilities $(\mathrm{p}=0.042, \mathrm{n}=44)$ (Table 3).

To test for a duration effect, we compared the change $(\Delta)$ from pre-treatment to post-treatment in participants who took Gene-Eden-VIR for less then two months and those who took Gene-Eden-VIR for two months or more. The results showed that participants who took GeneEden-VIR for the longer period reported a $220 \%$ larger decrease in their symptoms $(\mathrm{p}=0.044, \mathrm{n}=32)$ (Table 4).

We could not test for a dose effect since the number of participants who took 1,3 or 4 capsules per-day was too small for statistical analysis.

To test for a possible interviewer bias, we compared the answers to the pre-treatment questions collected by the American and the Israeli call centers. We also compared the answers to the post-treatment questions between the two call centers. In both cases, the difference between the answers was not statistically significant $(\mathrm{p}=$ $0.30, \mathrm{n}=154$, for pre-treatment, and $\mathrm{p}=0.36, \mathrm{n}=154$, for post-treatment, Table 5). This means that although the centers included different interviewers from different cultures working at different times of day, Americans working during the day and Israelis working during the night, the answers were similar. Hence, the results showed no interviewer bias. Similar results were obtained

Table 3. Pre-treatment vs. post-treatment, physical abilities, energy level, and mental abilities.

\begin{tabular}{ccccc}
\hline Question & N of P & Pre-T Score & Post-T Score & p Value \\
\hline Physical Abilities & 47 & 4.90 & 5.55 & $<0.001$ \\
Energy Level & 54 & 4.57 & 5.53 & $<0.001$ \\
Mental Abilities & 44 & 5.03 & 5.42 & 0.042 \\
\hline
\end{tabular}

* Statistical analysis was performed using a single score for each participant. The score was equal to the average of all answers in the relevant block of questions.

Table 4. Duration of treatment.

\begin{tabular}{cccc}
\hline Duration of Treatment & $\mathrm{N}_{\text {of }} \mathrm{P}^{*}$ & $\begin{array}{c}\text { Change }(\Delta) \text { from } \\
\text { Pre-T to Post- }\end{array}$ & Statistics \\
\hline Less than 2 Months & 13 & 0.43 & $\mathrm{p}=0.044$, \\
$\mathrm{n}=32$
\end{tabular}

*Statistical analysis was performed using the change in scores from pretreatment to post-treatment reported by present users only. The analysis used one score per participant. The score was equal to the average of the answers to questions B1 - B5. 
for the other sections of the questionnaire (physical abilities, energy, and mental abilities, data not shown) (Table 5).

To test for a possible selection bias, we compared the answers to the pre-treatment questions by the past and present users of Gene-Eden-VIR. We also compared the answers to the post-treatment questions between the two groups, and the change $(\Delta)$ from pre-treatment to posttreatment. In all three tests, the difference between the answers was not significant $(\mathrm{p}=0.18, \mathrm{n}=154$, for pretreatment, $\mathrm{p}=0.72, \mathrm{n}=154$, for post-treatment, and $\mathrm{p}=$ $0.47, \mathrm{n}=154$, for the change $(\Delta)$, Table 6). This means that, statistically, the answers by the present users are the same as the answers by the past users, and therefore, show no selection bias (Table 6).

An issue unique to natural products is the concern about the therapeutic consistency of marketed products. See discussion on this issue in the FDA guidelines for botanical New Drug Applications (NDA) [6]. To test the therapeutic consistency of Gene-Eden-VIR, we compared the two batches used by the participants. The capsules in these batches were produced at two different manufacturing sites, and completed about 10 months apart. The results showed that the answers given by the participants who used the capsules from Batch 1 were the same as those given by the participants who used the capsules from Batch $2(\mathrm{p}=0.988, \mathrm{n}=160$, Table 7). Hence, the results indicated that, although Gene-EdenVIR is a natural product, its formula has therapeutic consistency (Table 7).

\section{Discussion}

This post marketing clinical study showed that individu-

Table 5. USA vs. Israel call centers.

\begin{tabular}{cccccc}
\hline & \multicolumn{2}{c}{ USA } & \multicolumn{2}{c}{ Israel } & Statistics \\
\cline { 2 - 5 } & $\mathrm{N} \mathrm{of} \mathrm{A}^{*}$ & Score & N of A & Score & \\
\hline Pre-T & 81 & 3.03 & 73 & 2.74 & $\mathrm{p}=0.30, \mathrm{n}=154$ \\
Post- $\mathrm{T}$ & 81 & 5.04 & 73 & 5.36 & $\mathrm{p}=0.36, \mathrm{n}=154$ \\
\hline
\end{tabular}

*The data may include up to five answers per participant.

Table 6. Past vs. present users.

\begin{tabular}{cccccc}
\hline & \multicolumn{2}{c}{ Past } & \multicolumn{2}{c}{ Present } & Statistics \\
\cline { 2 - 5 } & N of A $^{*}$ & Score & N of A & Score & \\
\hline Pre-T & 46 & 3.17 & 108 & 2.77 & $\begin{array}{c}\mathrm{p}=0.18, \\
\mathrm{n}=154\end{array}$ \\
Post-T & 46 & 5.28 & 108 & 5.16 & $\begin{array}{c}\mathrm{p}=0.72, \\
\mathrm{n}=154 \\
\mathrm{p}=0.47, \\
\mathrm{n}=154\end{array}$ \\
\hline
\end{tabular}

${ }^{*} \mathrm{~N}$ of $\mathrm{A}$ is Number of Answers. The data may include up to five answers per participant.
Table 7. Batch 1 vs. Batch 2.

\begin{tabular}{cccc}
\hline Batch & N of A $^{*}$ & $\begin{array}{c}\text { Change ( } \boldsymbol{\Delta}) \text { from } \\
\text { Pre-T to Post-T }\end{array}$ & Statistics \\
\hline 1 & 81 & 2.20 & $\mathrm{p}=0.988$ \\
$\mathrm{n}=160$ \\
2 & 79 & 2.20 & \\
\hline
\end{tabular}

*Note that the data may include up to five answers per participant.

als infected with the HPV, EBV, HSV, HCMV, or HCV reported a safe decrease in their symptoms following treatment with Gene-Eden-VIR. The participants also reported an increase in their physical abilities, an increase in their energy level, an increase in their mental abilities, and an improvement in their general health.

The results are consistent. We observed a statistically significant decrease in the severity, duration, and frequency of symptoms. The results also showed a duration effect. Participants treated for two months or more reported a larger decrease in their symptoms compared to those treated for less then two months.

The results are robust. They showed no interviewer bias, no selection bias, and therapeutic consistency of the Gene-Eden-VIR formula under varying manufacturing conditions.

This post marketing clinical study does not include a placebo control, that is, it is not a double-blinded study. Placebo controlled studies are the gold standard in medical research in pre-marketing clinical studies. However, except in rare cases, post marketing studies do not use placebo controls. They use other controls recommended by the FDA.

The FDA guidance lists five types of controls for both pre marketing and post marketing studies: 1) Placebo Concurrent Control, 2) Pre-treatment Concurrent Control, 3) Dose-response Concurrent Control, 4) Active (Positive) Concurrent Control, 5) External Control (Including Historical Control). The External Control "can be a group of patients treated at an earlier time (historical control)" [25].

The Gene-Eden-VIR post marketing study is a change from baseline study that includes a pre-treatment concurrent control and a proxy for an historical control.

The results are not likely to be a placebo effect. The current predominant and well-proven theories on the placebo effect suggest that its main mechanisms are conditioned reflexes and patient expectations [26]. The GeneEden-VIR product literature does not mention the possibility of a change in symptoms, and specifically, the severity, duration, and frequency of symptoms. Hence, the participants in this study could not have been primed for, or expect the reported effects. This lack of conditioned reflexes and patient expectations minimizes the possibility of a placebo effect, and supports the possibility of a physiological effect. 
All participants who started the study completed it; therefore, the study has no follow-up bias.

It should be noted that although we tested for some biases, others are still possible, for instance, the non-responsive bias.

This study relies on patient reported outcomes (PROs). Past studies showed that PROs had a significant role in the development and evaluation of new medicines [27]. According to the FDA, PROs are a valid and valuable source for measuring the efficacy of new drugs. They are reliable enough to warrant an approval of a label claim for a new drug. From the years 1997 to 2002, the FDA approved 23 new drugs based on PRO endpoints only. They include six anti-migraine products $\left(\mathrm{Amerge}^{\circledR}, \mathrm{Ax}\right.$ $\mathrm{ert}^{\mathbb{B}}$ ), several anti-epileptics (Gabitril ${ }^{\mathbb{B}}, \mathrm{Keppra}^{\mathbb{B}}$ ), and a variety of other therapy classes $\left(\right.$ Tamiflu $^{\mathbb{B}}$, Relenza ${ }^{\mathbb{B}}$ ) [27].

The FDA regards this source of data as valid and valuable. The scientific community also believes that PROs are valid and useful. Many major journals published clinical studies that use patient reported outcomes. The trust of the FDA and the scientific community in PROs should convince the medical community, and specifically, doctors, to consider studies that use PROs when recommending medical treatments to their patients.

A possible limitation of this type of study is the subjective report of symptoms. One might argue that the participants' have evaluated the effect of Gene-Eden-VIR on symptoms, which are unrelated to their infection. To address this question, we compared the symptoms reported by the participants to the standard signs and symptoms reported in the literature ("Harrison's principles of internal medicine", 18th edition). The comparison clearly showed that the reported symptoms and the major standard symptoms of viral infection as found in the literature overlapped (data not shown).

The size of the study group is a major concern in clinical studies. A group that is too small may fail to show a positive effect of the treatment. In addition, a small group could also misrepresent the diversity in the population. The standard principle for multivariate behavioral research is at least 10 patients at endpoint per dependent measure [28]. This study included one endpoint dependent measure (the change in symptoms from pre-treatment to post-treatment). This study population included 60 individuals. Hence, the size of the study group in this study is adequate.

One might also question the reliability of the participants recall period due to the long duration of the period under investigation (up to 54 weeks). This study used a "then-test" method. This method, also known as the retrospective pre-test-post-test design method, asks participants at the post-test period to think back to the pre-test period and retrospectively rate their condition. The "re- sponse shift" is defined as the difference between the "pre-test" and the "then-test" ratings. Currently, the response shift is a well documented and extensively research phenomenon [29]. According to the literature on "response shifts", participants may alter their internal standards, values, or conceptualization of their quality of life when experiencing changes in health states. These response shifts can affect or distort the reported scores and undermine the credibility of the observed medical or psychosocial effects. Many studies reported that after participants experience an improvement in their health, a then-test tends to show a decrease in the initial assessment of the original level of well being.

Since this clinical study uses the "then-test" method, we tested for a possible response shift by comparing the answers to the pre-treatment question at less then two months and at two months or more. The results showed a statistically significant increase in the pre-treatment score over time. The results indicated that the participants experience a response shift, however, in the opposite direction from what was expected. This response shift suggests that the participants do not tend to exaggerate, but tend to forget how bad their symptoms were before taking Gene-Eden-VIR. The tendency to forget adds support to the statistical significance of the results in this study.

Our research also shows an important and unique development process that may influence future developments in medicine. Gene-Eden-VIR was formulated by analyzing thousands of scientific papers with Computer Intuition. The basic premise of the computer program is that every future event is preceded by hints, and that the key to predicting these events is recognizing the significance of these hints.

In 1996, the first author of this paper completed a prototype of a psycholinguistic-based data-mining program that analyzes scientific text and assigns a rating to all ideas found in the text. The higher the rating, the more it hints on future events.

The following is a description of one prospective application of Computer Intuition. In 1995, Frederiksen published a paper entitled: Diagnostic Imaging in Dental Implantology [30]. At the time, Frederiksen was one of the world leading experts on the subject. To test the predictive power of the Computer Intuition analysis, Almog and Heisler from the University of Rochester devised a test. They conducted a Medline search for papers published between 1980 and 1996 using keywords relevant to the subject of diagnostics, imaging, and dental implantology. The search identified 34 papers. The content of these papers was analyzed with Computer Intuition.

The analysis produced three ideas. Two ideas were identical to the main conclusions described in Frederiksen's paper. This, by itself, was an impressive achievement. By using Computer Intuition, Almog and Heisler 
duplicated the results of a world leading expert quickly and inexpensively. However, while it took Frederiksen decades to build his expertise, Almog and Heisler acquired similar expertise within weeks.

The third idea suggested a new technology. This technology was not mentioned in Frederiksen's paper. The three ideas were published in 1997 [7].

How predictive was the Computer Intuition analysis? In 2006, Almog, Frederiksen, and four colleagues, published a survey of the academic and commercial field of diagnostic imaging in oral implantology [31]. In their paper, they reported an interesting observation. Beginning in 2000, three years after the publication of the Computer Intuition paper, "numerous companies from technology-transfer and commercial standpoint have introduced technology platforms that offer planning and guidance systems to facilitate dental implant placement procedures", the same technology proposed by the third idea three years earlier. This observation confirms the Computer Intuition based prediction.

The scientists who developed Gene-Eden-VIR predicted that Gene-Eden-VIR will have an antiviral effect. This post marketing clinical study shows that, as predicted by the Computer Intuition analysis, individuals infected with viruses report a safe decrease in the severity, duration, and frequency of symptoms following treatment with Gene-Eden-VIR. As in the Almog and Heisler research, the results of this research confirm the Computer Intuition based prediction. The difference between the two studies is that Almog and Heisler predicted a new medical device. In this research, the scientists who developed Gene-Eden-VIR predicted the safety and efficacy of a new treatment. Both Computer Intuition based predictions were right.

\section{Conclusions}

The scientists who developed Gene-Eden-VIR used Computer Intuition to design a natural product that targets latent viruses in infected individuals. This post marketing clinical study showed that, as predicted, individuals infected with HPV, EBV, HSV, HCMV, and HCV reported a safe decrease in their symptoms following treatment with Gene-Eden-VIR. These results prove that Computer Intuition, a psycholinguistic-based data-mining program of scientific text, can predict the safety and effectiveness of medical treatments, and, therefore, has the potential to revolutionize the $\mathrm{R} \& \mathrm{D}$ process in the pharmaceutical industry.

Finally, this study showed that the natural product Gene-Eden-VIR safely and effectively decreases symptoms in individuals infected with the HPV, HSV, EBV, HCMV, and HCV. Therefore, health care practitioners should recommend Gene-Eden-VIR as a safe and effective antiviral treatment to individuals infected with these viruses.

\section{REFERENCES}

[1] M. A. Stanley, "Genital Human Papillomavirus Infections: Current and Prospective Therapies," Journal of General Virology, Vol. 93, No. 4, 2012, pp. 681-691. doi:10.1099/vir.0.039677-0

[2] E. Gershburg and J. S. Pagano, "Epstein-Barr Virus Infections: Prospects for Treatment," Journal of Antimicrobial Chemotherapy, Vol. 56, No. 2, 2005, pp. 277-281. doi:10.1093/jac/dki240

[3] L. A. Jensen, J. D. Hoehns and C. L. Squires, "Oral Antivirals for the Acute Treatment of Recurrent Herpes Labialis," Annals of Pharmacotherapy, Vol. 38, No. 4, 2004, pp. 705-709. doi:10.1345/aph.1D285

[4] G. Andrei, E. De Clercq and R. Snoeck, "Novel Inhibitors of Human CMV," Current Opinion in Investigational Drugs, Vol. 9, No. 2, 2008, pp. 132-145.

[5] J. M. Pawlotsky, "New Antiviral Agents for Hepatitis C," F1000 Biology Reports, Vol. 4, No. 5, 2012.

[6] US Department of Health and Human Services, Food and Drug Administration, Center for Drug Evaluation and Research (CDER), "Guidance for Industry. Botanical Drug Products," 2004.

http://www.fda.gov/downloads/Drugs/GuidanceComplian ceRegulatoryInformation/Guidances/ucm070491.pdf

[7] D. M. Almog and E. M. Heisler, "Computer Intuition: Guiding Scientific Research in Imaging and Oral Implantology," Journal of Dental Research, Vol. 76, No. 10, 1997, pp. 1684-1688. doi: $10.1177 / 00220345970760101001$

[8] A. Singal, S. Kaur, N. Tirkey and K. Chopra, "Green Tea Extract and Catechin Ameliorate Chronic Fatigue-Induced Oxidative Stress in Mice," Journal of Medicinal Food, Vol. 8, No. 1, 2005, pp. 47-52. doi:10.1089/jmf.2005.8.47

[9] S. Y. Lyu, J. Y. Rhim and W. B. Park, "Antiherpetic Activities of Flavonoids against Herpes Simplex Virus Type 1 (HSV-1) and Type 2 (HSV-2) in Vitro," Archives of Pharmacal Research, Vol. 28, No. 11, 2005, pp. 12931301. doi:10.1007/BF02978215

[10] L. K. Chang, T. T. Wei and Y. F. Chiu, "Inhibition of Epstein-Barr Virus Lytic Cycle by (-)-Epigallocatechin Gallate," Biochemical and Biophysical Research Communications, Vol. 301, No. 4, 2003, pp. 1062-1068. doi:10.1016/S0006-291X(03)00067-6

[11] L. C. Lin, Y. C. Kuo and C. J. Chou, "Anti-Herpes Simplex Virus Type-1 Flavonoids and a New Flavanone from the Root of Limonium sinense," Planta Medica, Vol. 66, No. 4, 2000, pp. 333-336. doi:10.1055/s-2000-8540

[12] Y. Iwase, Y. Takemura, M. Ju-ichi, et al., "Inhibitory Effect of Flavonoid Derivatives on Epstein-Barr Virus Activation and Two-Stage Carcinogenesis of Skin Tumors," Cancer Letters, Vol. 173, No. 2, 2001, pp. 105109. doi:10.1016/S0304-3835(01)00615-2

[13] B. Ozcelik, I. Orhan and G. Toker, "Antiviral and Antimicrobial Assessment of Some Selected Flavonoids," Zeitschrift für Naturforschung C: A Journal of Biosciences, 
Vol. 61, No. 9-10, 2006, pp. 632-638.

[14] A. Arena, G. Bisignano and B. Pavone, "Antiviral and Immunomodulatory Effect of a Lyophilized Extract of Capparis spinosa L. Buds," Phytotherapy Research, Vol. 22, No. 3, 2008, pp. 313-317. doi:10.1002/ptr.2313

[15] C. Fiore, M. Eisenhut and R. Krausse, "J Antiviral Effects of Glycyrrhiza Species," Phytotherapy Research, Vol. 22, No. 2, 2008, pp. 141-148. doi:10.1002/ptr.2295

[16] G. J. Kapadia, M. A. Azuine and H. Tokuda, "Inhibitory Effect of Herbal Remedies on 12-O-tetradecanoylphorbol13-Acetate-Promoted Epstein-Barr Virus Early Antigen Activation," Phytotherapy Research, Vol. 45, No. 3, 2002, pp. 213-220. doi:10.1006/phrs.2001.0936

[17] J. C. Lin, "Mechanism of Action of Glycyrrhizic Acid in Inhibition of Epstein-Barr Virus Replication in Vitro," Antiviral Research, Vol. 59, No. 1, 2003, pp. 41-47. doi:10.1016/S0166-3542(03)00030-5

[18] T. Sekizawa, K. Yanagi and Y. Itoyama, "Glycyrrhizin Increases Survival of Mice with Herpes Simplex Encephalitis," Acta Virologica, Vol. 45, No. 1, 2001, pp. 5154.

[19] F. Benencia and M. C. Courreges, "In Vitro and in Vivo Activity of Eugenol on Human Herpesvirus," Phytotherapy Research, Vol. 14, No. 7, 2000, pp. 495-500. doi:10.1002/1099-1573(200011)14:7<495::AID-PTR650 $>3.0 . \mathrm{CO} ; 2-8$

[20] Y. Tragoolpua and A. Jatisatienr, "Anti-Herpes Simplex Virus Activities of Eugenia caryophyllus (Spreng.) Bullock \& S. G. Harrison and Essential Oil, Eugenol," Phytotherapy Research, Vol. 21, No. 12, pp. 1153-1158. doi: $10.1002 /$ ptr.2226

[21] Y. Orihara, H. Hamamoto, H. Kasuga, et al., "A Silkworm-Baculovirus Model for Assessing the Therapeutic Effects of Antiviral Compounds: Characterization and Application to the Isolation of Antivirals from Traditional Medicines," Journal of General Virology, Vol. 89, No. 1, 2008, pp. 188-194. doi:10.1099/vir.0.83208-0

[22] S. W. Jian, C. E. Mei, Y. N. Liang, et al., "Influence of Selenium-Rich Rice on Transformation of Umbilical Blood B Lymphocytes by Epstein-Barr Virus and Epstein-Barr Virus Early Antigen Expression," Ai Zheng, Vol. 22, No. 1, 2003, pp. 26-29.

[23] H, Wojtowicz, K. Kloc, I. Maliszewska, et al., “Aza- analogues of Ebselen (Selenium-Containing Agents) as Antimicrobial and Antiviral Agents: Synthesis and Properties," Il Farmaco, Vol. 59, No. 11, 2004, pp. 863-868. doi:10.1016/i.farmac.2004.07.003

[24] G. N. Schrauzer, "Effects of Selenium and Low Levels of Lead on Mammary Tumor Development and Growth in MMTV-Infected Female Mice," Biological Trace Element Research, Vol. 125, No. 3. 2008, pp. 268-275. doi:10.1007/s12011-008-8172-1

[25] US Department of Health and Human Services, Food and Drug Administration, Center for Drug Evaluation and Research (CDER), "Guidance for Industry, E 10 Choice of Control Group and Related Issues in Clinical Trials," 2001.

http://www.fda.gov/downloads/RegulatoryInformation/G uidances/ucm129460.pdf

[26] M. Breidert and K. Hofbauer, "Placebo: Misunderstandings and Prejudices," Deutsches Ärzteblatt International, Vol. 106, No. 46, 2009, pp. 751-755.

[27] R. J. Willke, L. B. Burke and P. Erickson, "Measuring Treatment Impact: A Review of Patient-Reported Outcomes and Other Efficacy Endpoints in Approved Product Labels," Controlled Clinical Trials, Vol. 25, No. 6, 2004, pp. 535-552. doi:10.1016/j.cct.2004.09.003

[28] P. D. Harvey and R. S. Keefe, "Studies of Cognitive Change in Patients with Schizophrenia Following Novel Antipsychotic Treatment," American Journal of Psychiatry, Vol. 158, No. 2, 2001, pp. 176-184. doi:10.1176/appi.ajp.158.2.176

[29] C. E. Schwartz, R. Bode, N. Repucci, et al., "The Clinical Significance of Adaptation to Changing Health: A MetaAnalysis of Response Shift," Quality of Life Research, Vol. 15, No. 9, 2006, pp. 1533-1550. doi:10.1007/s11136-006-0025-9

[30] N. L. Frederiksen, "Diagnostic Imaging in Dental Implantology," Oral Surgery, Oral Medicine, Oral Pathology, Oral Radiology and Endodontology, Vol. 80, No. 5, 1995, pp. 540-554. doi:10.1016/S1079-2104(05)80153-2

[31] D. M. Almog, B. W. Benson, L. Wolfgang, et al., "Computerized Tomography-Based Imaging and Surgical Guidance in Oral Implantology," Journal of Oral Implantology, Vol. 32, Vol. 1, 2006, pp. 14-18. 